\title{
Incentivos al trabajo profesional docente y su relación con las políticas de evaluación e incentivo económico individual ${ }^{*}$
}

\author{
Professional teaching work incentives and their relation to assessment \\ and individual economic incentive policies \\ Incentivos para o trabalho profissional docente e sua relação com \\ as políticas de avaliação e incentivo financeiro individual
}

\author{
Felipe Acuña Ruz. ${ }^{a}$ \\ ${ }^{a}$ Centro de Investigación Avanzada en Educación, Universidad de Chile. Fono: 02-9782597. \\ Correo electrónico: facuna@ ciae.uchile.cl
}

\begin{abstract}
RESUMEN
El fortalecimiento de la profesión docente ha sido reconocido como fundamental para mejorar la calidad de la educación a nivel mundial. La lógica que la política educativa en Chile ha tomado para lograr este objetivo se sustenta en gran medida, en el diseño e implementación de programas de evaluación conducentes de forma directa o indirecta a incentivos económicos individuales. Sin embargo, ¿cuán alineados están los supuestos de estos programas con lo que realmente incentiva al profesorado a hacer bien su trabajo? La realización de cuatro grupos focales y seis entrevistas semi-estructuradas dan pie al principal hallazgo de este estudio: existen cuatro núcleos que incentivan el trabajo profesional docente con los cuales éstos atribuyen un sentido negativo, tanto a los programas de evaluación del desempeño, como a los incentivos económicos individuales. Estos resultados permiten discutir los supuestos economicistas y culturalistas que sustentan los programas de evaluación e incentivo económico en Chile.
\end{abstract}

Palabras clave: profesión docente, políticas de evaluación e incentivos económicos, cultura docente.

\begin{abstract}
Strengthening the teaching profession has been recognized as key to improving the quality of education worldwide. The logic that education policy in Chile has taken to achieve this goal is based largely on the design and implementation of assessment programs leading directly or indirectly to individual economical incentives. However, how aligned are the assumptions of these programs with what actually encourages teachers to do their job? The performance of four focus groups and six semi-structured interviews give rise to the main finding of this study: there are four cores that encourage teachers' professional job. With this, they attribute a negative sense to both performance evaluation programs and individual economic incentives programs. These results allow discussing the economistic and culturalist assumptions underlying assessment programs and economic incentives in Chile.
\end{abstract}

Key words: teaching profession, evaluation and economic incentives policies, teachers culture.

Esta investigación fue posible gracias al apoyo del Centro de Investigación Avanzada en Educación (PIA-CONICYT proyecto CIE-05) de la Universidad de Chile, y gracias a la investigación Subjetividad docente, malestar y condiciones de trabajo en profesores/as de Enseñanza Media de Santiago (2010-2012) financiada por la Vice-rectoría de Desarrollo e Investigación (VID) de la Universidad de Chile. 


\section{RESUMO}

O fortalecimento da profissão docente tem sido reconhecido como fundamental para melhorar a qualidade da educação em nível mundial. A lógica que a política educativa no Chile tem adotado para atingir este objetivo baseiase, em grande parte, em projeto e implementação de programas de avaliação que levam direta ou indiretamente aos incentivos financeiros individuais. Porém, quão alinhado estão os pressupostos de esses programas com o que realmente incentiva professores a fazer o seu trabalho? A realização de quatro grupos focais e seis entrevistas semiestruturadas permitiu-nos conhecer os principais achados de este estudo: há quatro núcleos incentivadores do trabalho profissional docente pelos quais os sujeitos atribuem um sentido negativo tanto aos programas de avaliação de desempenho como aos incentivos financeiros individuais. Resultados permitem discutir os pressupostos econômicos e culturais que sustentam os programas de avaliação de desempenho e incentivos financeiros no Chile.

Palavras chave: profissão docente, políticas de avaliação e incentivos financeiros, cultura docente.

\section{INTRODUCCIÓN}

Existen dos tendencias globales que han contribuido de forma significativa en publicitar al docente como un sujeto clave en el aseguramiento de la calidad educacional. La primera, basada en los principios de la justicia social y los derechos humanos, se visibiliza públicamente en Jomtien, Tailandia, el año 1990, bajo el nombre de Conferencia Mundial de Educación para Todos. Esta conferencia, convocada por UNESCO, genera un momento político significativo pues, tras el diagnóstico de la persistencia de realidades injustas que no permiten asegurar el derecho a la educación, se traza un objetivo global que consiste en asegurar las necesidades básicas de aprendizaje, es decir, la enseñanza primaria para todos los niños y niñas del mundo (UNESCO, 1990).

La segunda tendencia se visibiliza públicamente el mismo año 1990 y es conocida como el "Consenso de Washington". En éste, políticos de todo el continente americano llegan a acuerdo sobre el impacto positivo que para el crecimiento económico tiene una mayor disciplina fiscal, la liberalización de la política comercial y del régimen de inversiones, la desregulación de los mercados internos, y la privatización de las empresas estatales (Burki y Perry, 1998). Estas políticas de liberalización a escala global se han denominado neoliberalismo. El Banco Mundial es uno de los organismos internacionales que con mayor fuerza ha impulsado el neoliberalismo, y en esta cruzada ha entendido que la educación es un tema central, por lo que diseña sus propios marcos orientadores. En 1995 aparecen las Prioridades y estrategias para la educación, donde proponen seis reformas esenciales, entre las que destaca la atención a los resultados y su uso para establecer y vigilar las prioridades públicas y la inversión pública focalizada (Banco Mundial, 1996). Diez años más tarde, aparece Incentives to Improve Teaching. Lessons for Latin America, editado por Emiliana Vegas, donde se reflexiona en específico sobre qué incentivos son efectivos para mejorar la práctica docente con el objetivo de mejorar los resultados académicos de los estudiantes (Vegas, 2005).

Estas dos tendencias globales, la educación para todos y el neoliberalismo, pese a provenir de matrices ideológicas distintas y estar orientadas por principios diferentes, tienen un importante punto de encuentro: asegurar que los estudiantes consigan resultados de aprendizajes reconocidos y mesurables. Para llevar a cabo esta idea es clave fortalecer la profesión docente, y la forma en que se ha llevado a cabo esto en gran parte del mundo es mediante políticas de evaluación del desempeño, siendo una de las peculiaridades de Chile que éstas se han vinculado con programas de incentivo económico individual (Murillo, González y Rizo, 2006). 
Es en este contexto que emerge la necesidad de investigar con mayor profundidad cuán alineados están los supuestos de estas políticas, en el caso de Chile, con lo que realmente incentiva al profesorado a hacer bien su trabajo. Para abordar esta pregunta he realizado un estudio cualitativo, basado en grupos focales y entrevistas semi-estructuradas a docentes de enseñanza media. Antes de detallar los resultados, es importante vincular este contexto global al contexto local de las políticas de evaluación e incentivo en Chile.

\section{2. "BURÓCRATAS SIN INCENTIVOS": LA VISIÓN DE LOS DOCENTES CHILENOS A FINES DE LOS AÑOS 90}

El año 2000 es clave en Chile en su intento por asegurar que los estudiantes chilenos consigan aprendizajes reconocidos y mesurables. Dicho año sucede un hecho que permite articular dos lógicas centrales para comprender la realidad local de las políticas docentes: se conocen los resultados del Sistema Nacional de Medición de la Calidad de la Educación (SIMCE) de los estudiantes de cuarto básico, sobre quienes estaba la gran expectativa de ser la primera generación hija de la reforma educacional de 1996 (Cox, 2003; Belleï, 2001b; García-Huidobro, 2001). Por lo mismo, el estancamiento observado en los resultados obligó a buscar nuevas interpretaciones.

En primer lugar, un discurso fuerte utiliza la lógica culturalista para explicar el fenómeno. Este discurso es expuesto con claridad por Núñez $(2004,2003,1988)$ y por Belleï (2001a, 2001b), quienes sostienen que existen identidades y culturas docentes en tensión. Desde fines de los años ochenta, pero sobre todo en la década de los años noventa, lo que estaba en disputa a partir de la implementación de la reforma educacional eran dos matrices culturales distintas. Por un lado, estaba la cultura docente predominante, la cultura burocrática y funcionaria, la cual se basaba en un humanismo desvinculado de lo productivo con cierto enclaustramiento social y, por tanto, con un conservadurismo pedagógico. Estas características matrices son para Belleï las causas que explican que los docentes se sientan extraños a los cambios que la reforma escolar chilena ha intentado implementar, es decir, una reforma que busca fortalecer una cultura profesional, basada en una vinculación a lo productivo y la eficiencia tecnológica, con una apertura a la sociedad y el mundo y, por ende, con una pedagogía moderna (2001b).

En segundo lugar, emerge con fuerza un discurso que utiliza la lógica económica para explicar el problema de estancamiento en los resultados académicos de los estudiantes. Este discurso es expuesto con claridad por Mizala y Romaguera (2002a, 2002b). Estas autoras argumentan que los pobres resultados del SIMCE se deben, en gran medida, al bajo desempeño de los docentes. Este bajo desempeño se explica por la desmotivación y el decaimiento en el esfuerzo personal que generan los sueldos homogéneos entre ellos. El problema se relacionaba, en gran parte, con el Estatuto Docente (Ley N $\left.{ }^{\circ} 19.070\right)^{1}$, pues al haber repuesto elementos de una carrera burocrática no permitía diferenciar sueldos según desempeño. Así, un buen docente no tenía ningún incentivo para esforzarse más que su colega que era un mal docente. Una forma de remediar este problema era construir sistemas de evaluación del desempeño para distinguir entre "buenos" y "malos" docentes, y reconocer a los "buenos" a través de un incentivo económico.

Ley N 19.070. (1991). Estatuto de los Profesionales de la Educación. Santiago: Ministerio de Educación. 
De esta forma, la lógica culturalista le da cierta legitimidad histórica y sociológica a la lógica económica, toda vez que la reticencia de los docentes hacia la introducción de políticas de evaluación e incentivo económico individual se debe, fundamentalmente, a una cultura cuyos principios matrices se contradicen con la cultura profesional. El discurso contradictorio del profesorado, quienes, por un lado, rechazan las políticas de incentivo económico pues no resuelven realmente sus problemas esenciales de bajos sueldos y, por otro, niegan que los sueldos sean el motor de su profesión, es explicado por la lógica culturalista como evidencia de la presencia de los rasgos universales de la cultura docente burocrática y funcionaria que se alejan del productivismo y, a su vez, a la presencia de nuevos elementos de una cultura profesional que comienzan a operar de forma desarticulada (Belleï, 2001a; Beleï, Elgueta y Milesi, 1997).

Este argumento es integrado en los supuestos de la lógica económica. Desde esta lógica se empieza a señalar que si lo que se desea es fortalecer la profesión docente, se debe realizar un cambio cultural y la forma de realizar esto es vinculando el desempeño de los docentes a incentivos económicos (Mizala y Romaguera, 2002a, 2002b). Así, el camino para el diseño e implementación de políticas de evaluación e incentivo económico individual contó con la legitimidad de dos lógicas aparentemente en discusión, pero que resultaron dialogar bien. Quienes pensaban desde la lógica económica, comenzaron a interpretar los resultados de sus investigaciones desde una lógica culturalista. Como señalan Mizala y Romaguera, a propósito de la buena percepción del Sistema Nacional de Evaluación Docente (SNED) por parte de los profesores, "se ha estado produciendo en los últimos años un cambio en la cultura tradicional docente" (2002a: 30).

La Tabla N 1 sintetiza el problema y la solución con que ambas lógicas comprendieron el fenómeno social del desempeño y los incentivos al trabajo profesional docente:

Tabla $N^{\circ} 1$. Lógicas para comprender el "problema docente" a fines de los años 90

\begin{tabular}{|l|l|l|}
\hline \multicolumn{1}{|c|}{ Lógica económica } & \multicolumn{1}{c|}{ Lógica cultural } \\
\hline Problema & $\begin{array}{l}\text { Sueldos homogéneos que desmotivan y } \\
\text { no estimulan a los mejores docentes a } \\
\text { esforzarse. }\end{array}$ & $\begin{array}{l}\text { Cultura burocrática funcionaria que se } \\
\text { asocia a un humanismo desvinculado de } \\
\text { lo productivo. }\end{array}$ \\
\hline Solución & $\begin{array}{l}\text { Identificar a los mejores y reconocer su } \\
\text { esfuerzo mediante un incentivo } \\
\text { económico. }\end{array}$ & $\begin{array}{l}\text { Fortalecer la cultura profesional que se } \\
\text { asocia a la búsqueda de interés } \\
\text { instrumental. }\end{array}$ \\
\hline
\end{tabular}

Fuente: elaboración propia.

Así, desde 1996 a la fecha se ha generado una serie de dispositivos orientada a fortalecer la profesión docente: en 1996 se crea el SNED, el año siguiente el Premio a la Excelencia Pedagógica, el 2000 la Asignación de Excelencia Pedagógica (AEP), el 2003 el Sistema Nacional de Evaluación del Desempeño Profesional Docente (Evaluación Docente), el 2004 la Asignación Variable por Desempeño Individual (AVDI), el 2006 la prueba INICIA y el 
2011 la Beca Vocación de Profesor o ramas como la prueba INICIA². Todas estas políticas evidencian que para la política educativa chilena la forma de fortalecer la profesión docente es evaluando el desempeño y vinculando este desempeño a incentivos económicos individuales. Frente a esta lógica, el Colegio de Profesores de Chile ha reaccionado con un público rechazo:

La Carrera debe poner fin a los estímulos individuales y competitivos que constituyen la Asignación de Excelencia Pedagógica (AEP) y el AVDI (Asignación Voluntaria de Desempeño Individual). No debieran existir estímulos económicos fuera de la carrera, tampoco se justifica su permanencia dentro de la Carrera, ya que lo que se ha planteado es que ésta debe ser articulada al sistema de evaluación docente, el que debe ser corregido (Colegio de Profesores, 2010: 4).

Junto con rechazar los incentivos económicos individuales, en el mismo documento (Colegio de Profesores, 2010) manifiestan su descontento con la Evaluación Docente, la cual, según indican, debe ser corregida y perfeccionada.

No sólo los docentes han cuestionado estas políticas y programas, sino que intelectuales internacionales señalan que los principios de éstas, al estar basadas en el fundamentalismo de mercado y en una identidad emprendedora-competitiva (Hargreaves, 2005b; Day, 2002), se contraponen a los rasgos constitutivos de la cultura docente. El argumento es que estas políticas ignoran y con ello erosionan el sentido identitario del trabajo docente. Por ello Day sostiene: "paradojalmente, entonces, las reformas impuestas pueden en el largo plazo disminuir la capacidad de los docentes de elevar los estándares" (2002: 686).

La identidad y la cultura docente son elementos claves a considerar para que una reforma o política educativa tenga efecto, por lo mismo, cuando una política no considera estas dimensiones puede erosionar o desestabilizar la percepción de la labor que dota de sentido a la identidad profesional y, con ello, perjudicar el buen desempeño laboral (Kelchtermans, 2005; Day, 2002). El problema, como señala Kelchtermans, es:

Si los cambios demandados requieren abandonar formas de hacer que reflejan creencias y normas profundamente sostenidas sobre la buena enseñanza (percepción de la labor), la reforma implícita o explícitamente cuestiona la autoestima del docente y provocará intensas emociones de dudas, ansiedad, culpa, vergüenza (2005: 1001).

Así, comprender la noción de buena enseñanza o la percepción de la labor se vuelve un elemento relevante para estudiar qué elementos están en juego a la hora de atribuir sentido a las reformas, políticas o cambios educacionales que se buscan implementar. Sobre todo si se considera que la profesión docente vive un momento histórico en que existe una intensificación, sobreregulación y control de su tarea laboral (Day y Qu, 2012; Ávalos, Cavada, Pardo y Sotomayor, 2010; Hargreaves, 2005a).

En este sentido, aún son escasas las investigaciones nacionales que han estudiado el impacto que estas políticas tienen en la configuración de la identidad de los docentes. Autores

El SNED es un incentivo económico colectivo asociado indirectamente al desempeño de los docentes. La AEP es una evaluación voluntaria, la Evaluación Docente es una evaluación obligatoria, el AVDI es una evaluación voluntaria. Los resultados del SNED, la AEP y los resultados de la Evaluación Docente / AVDI posibilitan a los docentes llegar a obtener incentivos económicos individuales que pueden ir hasta un treceavo sueldo mensual (Bravo, Falck, González, Manzi y Peirano, 2009). La prueba INICIA es una prueba para egresados de las carreras de pedagogía, y la Beca Vocación de Profesor es una incentivo para estimular a estudiantes a ingresar a estudiar pedagogía. 
como Sisto (2012) concluyen que los docentes resisten estas políticas, al describirlas como limitadas y subjetivas.

En base a lo expuesto, la pregunta que orienta la investigación es: ¿qué tan alineados están los supuestos de los programas de evaluación del desempeño y de incentivos económicos individuales con lo que realmente incentiva el trabajo de los docentes? Esta pregunta se traduce en dos objetivos específicos: i) identificar y describir los núcleos con los cuales los docentes atribuyen sentidos a los programas de evaluación del desempeño profesional y a los programas de incentivos económicos individuales, e; ii) identificar y describir los elementos que los docentes consideran relevantes para la buena realización de su labor.

\section{METODOLOGÍA DE TRABAJO}

Éste es un estudio exploratorio basado en metodología cualitativa. He indagando en el orden del decir en dos niveles: el discurso público y el discurso privado a través de cuatro grupos focales y seis entrevistas semi-estructuradas, respectivamente. La muestra intencionada (Miles \& Huberman, 1994) está compuesta por dos criterios: el nivel de bienestar que perciben los docentes (Cornejo, 2009), y los resultados en la evaluación de desempeño profesional. Estos criterios configuran una muestra estructurada basada en cuatro perfiles hipotéticos de docentes (ver Tabla $\mathrm{N}^{\mathrm{o}} 2$ ), cada uno construyendo, posiblemente, un discurso distinto respecto a las políticas de evaluación del desempeño e incentivos económicos individuales:

Tabla $N^{o}$ 2. Perfiles docentes

\begin{tabular}{|l|c|c|}
\hline & $\begin{array}{c}\text { Docentes evaluados como } \\
\text { básicos }\end{array}$ & $\begin{array}{c}\text { Docentes evaluados como } \\
\text { competentes o destacados }\end{array}$ \\
\hline Bajo nivel de bienestar & Perfil 1 & Perfil 2 \\
\hline Alto nivel de bienestar & Perfil 3 & Perfil 4 \\
\hline
\end{tabular}

Fuente: elaboración propia.

El trabajo en terreno se realizó en cinco establecimientos de la Región Metropolitana entre los meses de septiembre y diciembre de 2010. En el marco de la investigación Subjetividad docente, malestar y condiciones de trabajo en profesores/as de Enseñanza Media de Santiago ${ }^{3}$ (2010-2012) se utilizó un estudio cuantitativo anterior sobre salud laboral (Cornejo, 2009) para abordar los criterios de bienestar y malestar. Las 45 escuelas que fueron parte de dicho estudio fueron ordenadas según su puntaje en el Índice General

Investigación financiada por la Vice-rectoría de Desarrollo e Investigación (VID) de la Universidad de Chile, coordinada por Rodrigo Cornejo. 
de Bienestar. Las escuelas de los extremos fueron consideradas escuelas con alto y bajo nivel de bienestar, realizándose dos grupos focales en escuelas con alto nivel de bienestar y dos grupos focales en escuelas con bajo nivel de bienestar. No fue posible incluir en los grupos focales el criterio de nivel de desempeño, por lo que en ellos sólo se construyeron dos perfiles de docentes: bajo bienestar (perfil 1-2) y alto bienestar (perfil 3-4). El criterio de nivel de desempeño se pudo resolver de forma satisfactoria en las entrevistas, realizándose dos entrevistas a docentes evaluados básicos, uno de cada nivel de bienestar, y cuatro entrevistas a docentes evaluados como competentes o destacados, dos por cada nivel de bienestar.

El tipo de análisis realizado se basa en la teoría fundamentada (Strauss y Corbin, 2002). Luego de realizar una codificación abierta, en que he sistematizado y analizado la información más relevante para cada perfil docente, identifiqué los códigos axiales o ejes centrales. Dentro de los códigos axiales, he identificado y seleccionado como relevantes cuatro grandes códigos de oposición, esto es, códigos que operan como distinciones enfrentadas al interior del discurso de los docentes (Miles \& Huberman, 1994). Finalmente, en base a estos códigos de oposición, construí mapas conceptuales siguiendo la lógica de las estructuras cruzadas y paralelas (Hébert, 2011; Martinic, 1992). A continuación presento los códigos de oposición y los mapas conceptuales.

\section{RESULTADOS}

\subsection{CÓDIGOS DE OPOSICIÓN}

En el discurso de los docentes abundan las distinciones enfrentadas. He escogido cuatro que permiten agrupar abundante información que los docentes producen en su discurso.

\subsubsection{Reconocimiento social/incentivos personales}

En una entrevista, una docente del perfil cuatro construye una distinción clave, y que a su vez emerge en el resto de los perfiles: ella señala de forma clara que reconocimiento e incentivos no son lo mismo.

"A ver, incentivar y reconocer son dos cosas distintas. A mí me gustaría que se me pagara más. A mí me encanta elaborar material, me encanta elaborar pruebas, guías, pero bien hechas, pero resulta que me veo corta de tiempo porque trabajo en dos partes [...]. Si tú me pagaras todo lo que gano en las otras partes [...]. Para mí el sueldo es una cuestión súper importante, entonces eso sería lo que a mí me gustaría. Ahora, reconocimiento, yo creo que, bueno, que de repente te palmoteen la espalda, y te digan «¿sabí qué? Lo estai haciendo súper». No estoy hablando que allí haya un bono" (María, entrevista, perfil 4).

Profundizando en esta distinción es posible identificar dos sub-códigos. Primero está el reconocimiento social que incluye referencias como la relevancia que los docentes otorgan a los actores cercanos (estudiantes, colegas y directivos) como aquellos muy validados para evaluar su desempeño profesional. Estos actores cercanos serían agentes claves en el mejoramiento profesional, pues son los verdaderos pares. Junto con ello, es posible incluir 
referencias que apelan a que actores más lejanos (sostenedor, Ministerio de Educación e incluso la misma sociedad, vía medios de comunicación) reconozcan la importancia del trabajo docente.

Un tercer conjunto de nociones apelan a que los docentes puedan tener mayores responsabilidades en la propia carrera profesional. Esta idea emerge con fuerza en los perfiles tres y cuatro, aquellos con alto bienestar, por ejemplo, en la idea de poder acceder a jefaturas dentro del departamento en que un docente trabaja.

Por último, es posible incluir un conjunto de referencias que apelan a tener mejores sueldos en dos sentidos distintos. Por un lado, los cuatro perfiles mencionan de forma redundante la idea de que el sueldo que tienen los docentes es injusto en comparación a otros profesionales como los médicos o los ingenieros. Por otro lado, está la idea sustentada por los perfiles dos, tres y cuatro, de que el sueldo no es digno en relación a la complejidad de la labor profesional que realizan. Nuevamente la comparación es con otras profesiones, enfatizándose que la docencia es una profesión muy compleja, y por ende, merece un sueldo digno a la complejidad del trabajo.

El segundo sub-código presente en esta distinción es incentivos personales. Este código agrupa dos conjuntos de referencias, primero, la idea de los perfiles dos y cuatro, aquellos con desempeño destacado, de que el trabajo docente precisa de un buen sueldo. Un buen sueldo se refiere a aquel capaz de satisfacer las necesidades personales de cada docente según sus propios intereses. Por ello, en una entrevista, un docente de perfil dos señala que un incentivo de cien mil pesos al mes es impactante para él, o como señala también en una entrevista una docente del perfil cuatro, un buen incentivo para ella equivale a lo que gana trabajando en dos colegios, haciendo preuniversitario los sábados y clases particulares los domingos.

En segundo lugar, se pueden incluir las referencias sobre el acceso a bienes para el fortalecimiento del trabajo pedagógico. Es posible agrupar estos bienes en tres tipos: i) acceso a materiales como libros, entrevistas o documentales de televisión u objetos tecnológicos como pendrives o notebooks; ii) acceso a espacios pedagógicos internos, como una "sala temática", donde el docente pueda invitar a los alumnos a un espacio ambientado especialmente para el trabajo de lenguaje o matemáticas, o el acceso a espacios pedagógicos externos como el teatro o el cine, y; iii) acceso a estudios de postgrado como posibilidad de actualización y perfeccionamiento. Si bien no todos los perfiles identifican cada uno de estos tres elementos, todos tienen alguno de ellos, es decir, algún bien que fortalezca su trabajo pedagógico.

\subsubsection{Incentivos como profesor / incentivos como trabajador}

Cuando los docentes describen potenciales incentivos, emerge una distinción entre incentivos e incentivos como profesor. En una entrevista, una docente del perfil dos elabora esta distinción con mucha claridad.

\footnotetext{
"Yo creo que a nosotros como docentes nos incentivaría más que cada vez que tú estás haciendo tu práctica hubiese personas preocupadas de decirte «lo estás haciendo bien, más o menos, cambia esto, cambia esto otro». Algo más cotidiano, porque ese incentivo [AVDI], claro, es dinero, pero las autoridades, en realidad, uno siente que no están preocupadas tanto de incentivarte como profesor" (Susana, entrevista, perfil 2).
} 
El primer sub-código de esta distinción es un código en vivo, pues utiliza la misma "etnocategoría" que utiliza la docente: incentivos como profesor. Éste permite agrupar referencias sobre aquellos elementos que tendrían un efecto positivo en el trabajo cotidiano del profesor, es decir, sobre el mejoramiento profesional. Es posible incluir nuevamente las referencias sobre lo importante que es que actores cercanos manifiesten su opinión sobre el propio quehacer del docente, y que exista un reconocimiento de las autoridades más lejanas. Junto con ello, es posible incluir las referencias sobre un sueldo digno, en el sentido que dé cuenta de lo complejo del trabajo como profesor. No obstante, a diferencia del reconocimiento social, se incluyen referencias sobre el acceso al perfeccionamiento o estudios de postgrado, el acceso a materiales y el acceso a espacios pedagógicos. Los incentivos como profesor son incentivos profesionales específicos para quienes se dedican al oficio de la docencia. Por ello, es en este sub-código donde se pueden agrupar las referencias, negativas o positivas, que hablan de la evaluación docente, pues ésta estaría relacionada al fortalecimiento profesional.

El segundo sub-código, referido en el fragmento como incentivos a secas por la docente, se aclara al revisar con cuidado otro fragmento expuesto por un docente en un grupo focal de alto bienestar:

"[...] o sea una persona que por el trabajo que entrega no perciba, por ejemplo, una remuneración de acuerdo, o sea es un trabajo, por mucha vocación que tengas por ejemplo, es un trabajo. Entonces partiendo de la base de que tú trabajas en base a motivar, o sea ¿tú cómo vas a motivar si tú no estás motivado?" (Jorge, grupo focal, alto bienestar perfil 3-4).

Se observa que, a diferencia de los incentivos como profesor, los incentivos como trabajador, como puede denominarse este segundo sub-código, son generales para cualquier persona que realiza un trabajo y que debe percibir una remuneración de acuerdo al trabajo realizado, es decir, se relacionan específicamente a las referencias que hablan del mejoramiento salarial. Entendidos así, es posible agrupar dos conjuntos de referencias relacionados al sueldo que he identificado anteriormente. Primero, contar con un buen sueldo, adecuado a las necesidades personales que posee el docente. Segundo, poseer un sueldo justo, es decir, acorde al sueldo que los pares profesionales, tales como los doctores o los ingenieros, reciben en comparación con los docentes.

\subsubsection{Vocación de salvador / vocación profesional}

Cuando los docentes se refieren a su percepción de la buena realización de su labor emerge una distinción donde se reitera el concepto de vocación. Esto no es casualidad, sino que en ambos sub-códigos se reconoce una motivación profunda por enseñar. La diferencia recae en que, el primer sub-código denominado vocación de salvador, es una vocación de entrega por el otro, en este caso por los estudiantes, sin importar ni pedir recompensas, y que se enfoca específicamente en salvar a unos cuantos estudiantes de su terrible realidad, mientras que, el segundo sub-código denominado vocación de profesional, es la entrega a la realización de un trabajo profesional de la mejor forma posible y con todas las responsabilidades que ello implica, pero también con las recompensas propias de cualquier trabajo profesional, y se enfoca en enseñar a la mayoría del curso, no sólo a unos pocos. Los siguientes fragmentos aclaran la distinción: 
“¿Qué incentivo tengo yo? Con ellos [la Corporación Municipal] no tengo incentivo. Pero mis alumnos sí, por ellos yo estoy aquí, si po'. Ése es mi incentivo, yo con que salve a tres o cuatro, es rico" (Rubén, entrevista, perfil 1).

"Mira, la verdad es que yo nunca he sentido que poco menos que es un apostolado, no, nunca. Ni lo voy a sentir tampoco. Para mí es una profesión más" (Marcela, entrevista, perfil 4).

El eje vocación de salvador es encarnado de forma ejemplar por la entrevista del perfil $1 \mathrm{y}$, en menor medida, por los grupos focales de bajo bienestar (perfil 1 y 2). En este eje es posible agrupar aquellas referencias que indican que el trabajo docente es una lucha donde se juega la vida y, por lo mismo, va más allá del dinero: "no se está acá por la plata". Además de esto, es posible incluir las referencias que indican que un buen docente es aquel que logra "salvar uno, dos o tres chiquillos", donde la salvación significa ser capaz de sacar a esos "chiquillos" de su contexto de marginalidad. Esto permite comprender el gran recelo que sienten a ser evaluados por alguien externo que no tenga la misma vocación ni trabaje en el mismo contexto, pues su juicio se torna irreal.

El eje vocación de profesional es encarnado de forma ejemplar por las entrevistas del perfil 4 y, en menor medida, por los grupos focales de alto bienestar (perfil 3 y 4). En este eje es posible agrupar un conjunto de referencias que señalan que el foco central del trabajo docente está en conseguir que los alumnos aprendan para que tengan las mismas oportunidades que otros alumnos más favorecidos socioeconómicamente. Junto con ello, es posible incluir aquellas referencias que indican que un buen docente apuesta por la movilidad social de todos sus estudiantes y no sólo de algunos, marcando una diferencia con el perfil uno. Esto convierte a la docencia en un trabajo profesional complejo, que es importante que sea evaluado y retroalimentado constantemente y de diversas formas, preferiblemente entre pares pero también por agentes externos, pues entre mayor claridad se tenga sobre cómo lograr aprendizajes, mejor se realizará la tarea de entregar oportunidades de movilidad social a los estudiantes.

\subsubsection{Formación en valores / formación en aprendizajes}

Esta última distinción se compone de sub-códigos que especifican cómo llevar a cabo la vocación docente. Por un lado, está la formación en valores, que quiere decir poner como foco de la enseñanza los valores, la enseñanza de ser buenas personas. Por otro lado, está la formación en aprendizajes, que quiere decir poner como foco de la enseñanza el aprendizaje significativo para la vida del estudiante. Los siguientes fragmentos aclaran la distinción:

"Eso es importante, un logro, uno trata de que los cabros... meter la familia, meter la familia. Cuando hay reunión de apoderados, o sea aquí hacen cada dos meses una reunión, yo hago todos los meses, me junto con los apoderados, con los pocos y nada que vienen, les seguimos dando valores, valores, valores, [para] sacarlos [de su contexto]" (Rubén, entrevista, perfil 1).

"[El buen docente] es comprometido con los alumnos, comprometido en su aprendizaje, en su aprendizaje, en su aprendizaje significativo digamos, porque se usa mucho, está muy manoseada la palabra aprendizaje significativo, pero no po', la idea es que realmente lo logren, y que te preocupes de la mayoría de los alumnos" (María, entrevista, perfil 4). 
El discurso de la entrevista del perfil uno, de un docente con bajo bienestar y nivel de desempeño básico, apuesta de manera reiterada en la formación en valores, mientras que las entrevistas del perfil cuatro, de docentes con alto bienestar y nivel de desempeño destacado, apuestan de manera reiterada en la formación en aprendizajes. Los discursos de estos perfiles vienen a ocupar la posición de tipos ideales de estos códigos axiales.

\subsection{MAPAS CONCEPTUALES}

El desafío de los mapas conceptuales es tornar más inteligibles los códigos de oposición recién expuestos. He construido tres mapas conceptuales. En el primero, he seguido la lógica de una estructura cruzada (Martinic, 1992), esto es, he cruzado los dos primeros códigos de oposición. Esto genera cuatro realidades teóricas. En el segundo, he utilizado la lógica de una estructura paralela, con los dos últimos códigos de oposición, al estilo del cuadro de Greimas (Hébert, 2011), evidenciando elementos de disyunción y conjunción en el discurso. El tercer mapa es una propuesta de articulación de ambos mapas.

El mapa conceptual $\mathrm{N}^{\circ} 1$ lo he construido cruzando el código de oposición incentivos como trabajador / incentivos como profesor, que he posicionado en el eje de las x, y el código de oposición reconocimiento social / incentivos personales, que he posicionado en el eje de las y.

Mapa conceptual $N^{o} 1$. Núcleos de sentido sobre los incentivos al buen desempeño profesional ${ }^{4}$

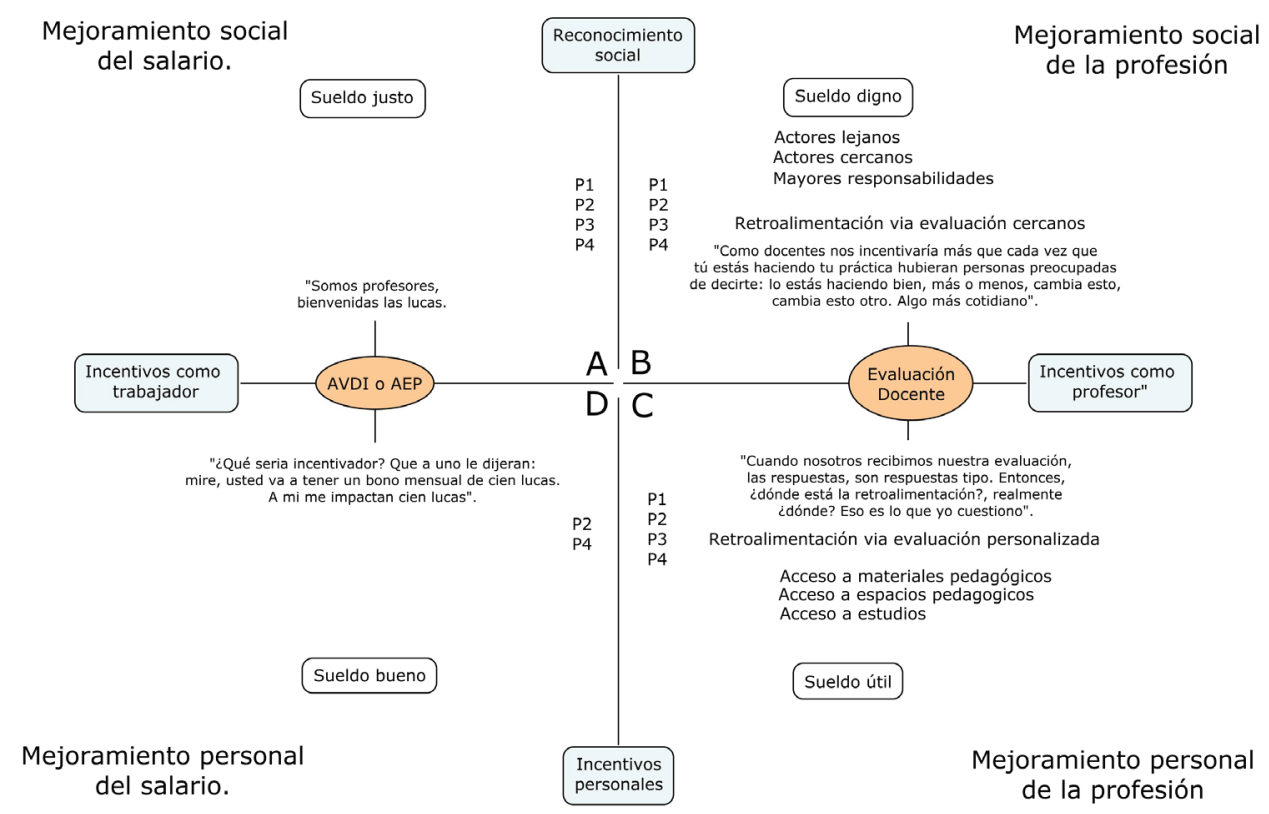

Fuente: elaboración propia.

$4 \quad$ P1: perfil de docentes uno; P2: perfil de docentes dos; P3: perfil de docentes tres; P4: perfil de docentes cuatro. 
El primer núcleo de sentido es el mejoramiento social del salario ubicado en la casilla A. Este núcleo es utilizado por los cuatro perfiles de docentes para atribuir un sentido negativo a los programas de incentivos económicos individuales, no criticando su lógica, sino indicando que dado que los salarios de los docentes son tan bajos en comparación a otras profesiones, un ingreso extra es bienvenido. El problema es que el ingreso extra es muy bajo. Así, por ejemplo, el AVDI se interpreta como un incentivo compensatorio aún escaso para restablecer la justicia que debiera existir en términos salariales entre profesores y sus pares de comparación más frecuentes: médicos e ingenieros.

El segundo núcleo de sentido es el mejoramiento social de la profesión, ubicado en la casilla B, con el cual los cuatro perfiles de docentes atribuyen un sentido negativo a los programas de evaluación del desempeño. En este núcleo es clave que actores cercanos retroalimenten el quehacer docente por medio de la evaluación del desempeño. El actual sistema de evaluación docente no involucra de manera directa el trabajo con pares cercanos (colegas o directores) o con estudiantes de la misma escuela, tampoco resulta en mayores responsabilidades al interior de la escuela, sino que se incluye un evaluador par que es cuestionado en su idoneidad. Por todo lo anterior, la evaluación docente se significa de forma negativa desde este núcleo de sentido.

El tercer núcleo de sentido es el mejoramiento personal de la profesión, ubicado en la casilla $\mathrm{C}$, con el cual los cuatro perfiles de docentes atribuyen igualmente un sentido negativo a los programas de evaluación del desempeño, pues aquí se espera de un programa de evaluación que sirva para retroalimentar la práctica personal docente, indicando cuáles fueron las principales fortalezas y debilidades. Existe una crítica transversal a los resultados de la evaluación docente en todos los perfiles, pues la evaluación docente, al entregar respuestas tipo como informes de evaluación, genera en los docentes frustración, desdén e incluso la sensación de que se les falta el respeto, pues se diluye todo lo "personal" o propio que debieran mejorar, en una respuesta estándar que no distingue ni por subsector ni por nivel de enseñanza.

Por último, el cuarto núcleo de sentido es el mejoramiento personal del salario, con el cual sólo los docentes del perfil dos y cuatro atribuyen un sentido negativo a los programas de incentivo económico individual debido a lo escaso de los mismos. Eso sí, las referencias de los docentes se confunden entre este núcleo de sentido y el núcleo $\mathrm{A}$. En este núcleo emerge la demanda por un buen sueldo con el objeto de poder realizar el proyecto de vida (profesional, familiar, etc.) de cada docente. La pretensión de un buen sueldo se vincula con el interés personal de viabilizar el proyecto de vida. Ejemplo de lo anterior es dejar de trabajar horas extras para concentrar todos los esfuerzos en un solo establecimiento. De esta forma, el problema no es el AVDI, sino lo escaso del premio que no impacta en las pretensiones de buen sueldo que los docentes de los perfiles dos y cuatro poseen.

Un hallazgo interesante que emerge de este mapa es que los perfiles uno y tres, aquellos con nivel de desempeño básico, no refieren en sus discursos elementos relacionados al núcleo mejoramiento personal del salario. Esto puede profundizarse al indagar en los dos siguientes códigos de oposición que refieren a la percepción de la buena realización de su labor. El mapa conceptual $\mathrm{N}^{\mathrm{o}}$ 2, utilizando la forma de una estructura paralela, me permite analizar el término complejo percepción de la buena realización de la labor, que se compone de dos sub-códigos que están en disyunción: vocación de salvador y vocación profesional. A su vez, estos sub-códigos están en una relación de conjunción y contradicción con los sub-códigos formar en valores y formar en aprendizajes. Lo anterior 
se puede representar de la siguiente forma:

Mapa conceptual $N^{o}$ 2. Percepción de la buena realización de la labor ${ }^{5}$

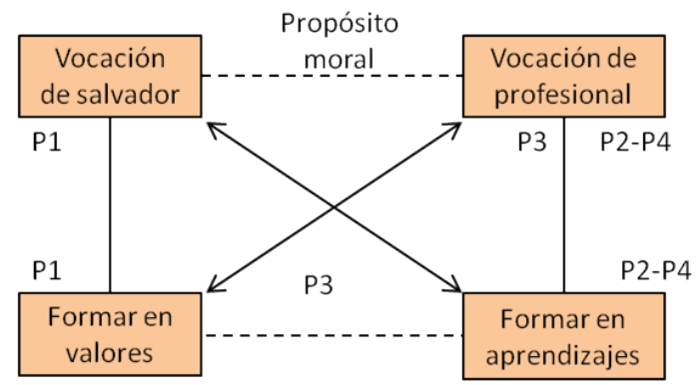

Fuente: elaboración propia.

La lectura de este mapa conceptual es: el término complejo percepción de la buena realización de la labor se compone de dos sub-códigos centrales: vocación de salvador que está en una relación de disyunción con vocación de profesional. Esta disyunción se expresa en que el código vocación de salvador está en una relación de contradicción con el código formar en aprendizajes, y este último, a su vez, está en relación de complementariedad con el código vocación de profesional. Por su parte, el sub-código vocación de profesional está en una relación de contradicción con el código formar en valores, y este último, a su vez, está en relación de complementariedad con el código vocación de salvador.

Así, el discurso de los docentes sobre la percepción de la buena realización de su labor no es homogéneo, sino que tiene importantes matices. Hay quienes se auto-perciben como buenos docentes en tanto son salvadores y para ellos lo fundamental de su trabajo es la entrega de valores (perfil 1). Otros se auto-perciben como buenos docentes en disyunción a esta imagen, y rehúsan ser apóstoles, héroes o salvadores. Dicen que su trabajo es un trabajo profesional, como cualquier otro, y que se fundamenta en generar aprendizajes, lo que por cierto incluye valores, pero esto no es lo prioritario para ellos (perfil 2 y 4). Junto con éstos, existen discursos que poseen elementos más cercanos a la auto-percepción de profesional, pero, a su vez, con una orientación en valores (perfil 3).

Lo interesante de este mapa conceptual es que profundiza la visión que los docentes tienen de su trabajo profesional, de lo que significa para ellos y ellas ser un buen docente. Se aprecia que no existe un discurso homogéneo frente a esto, sino que existen diferencias importantes que permiten hacer mucho más inteligible el mapa conceptual $\mathrm{N}^{\mathrm{o}} 1$. En efecto, dado que esta estructura paralela (mapa $\mathrm{N}^{\mathrm{o}} 2$ ) refiere a la percepción del trabajo en tanto docente y no en tanto trabajador, permite enriquecer el sector oeste del mapa conceptual $\mathrm{N}^{\circ}$ 1, es decir, donde están los núcleos mejoramiento social y personal de la profesión. Esto me permite construir un nuevo mapa que integra los dos anteriores: 
Estudios Pedagógicos XLI, N 1: 7-26, 2015

INCENTIVOS AL TRABAJO PROFESIONAL DOCENTE Y SU RELACIÓN CON LAS POLÍTICAS DE EVALUACIÓN E INCENTIVO ECONÓMICO INDIVIDUAL

Mapa conceptual $N^{o} 3$. Diferencias en los incentivos al buen desempeño profesional según la percepción de la buena realización de la labor ${ }^{6}$

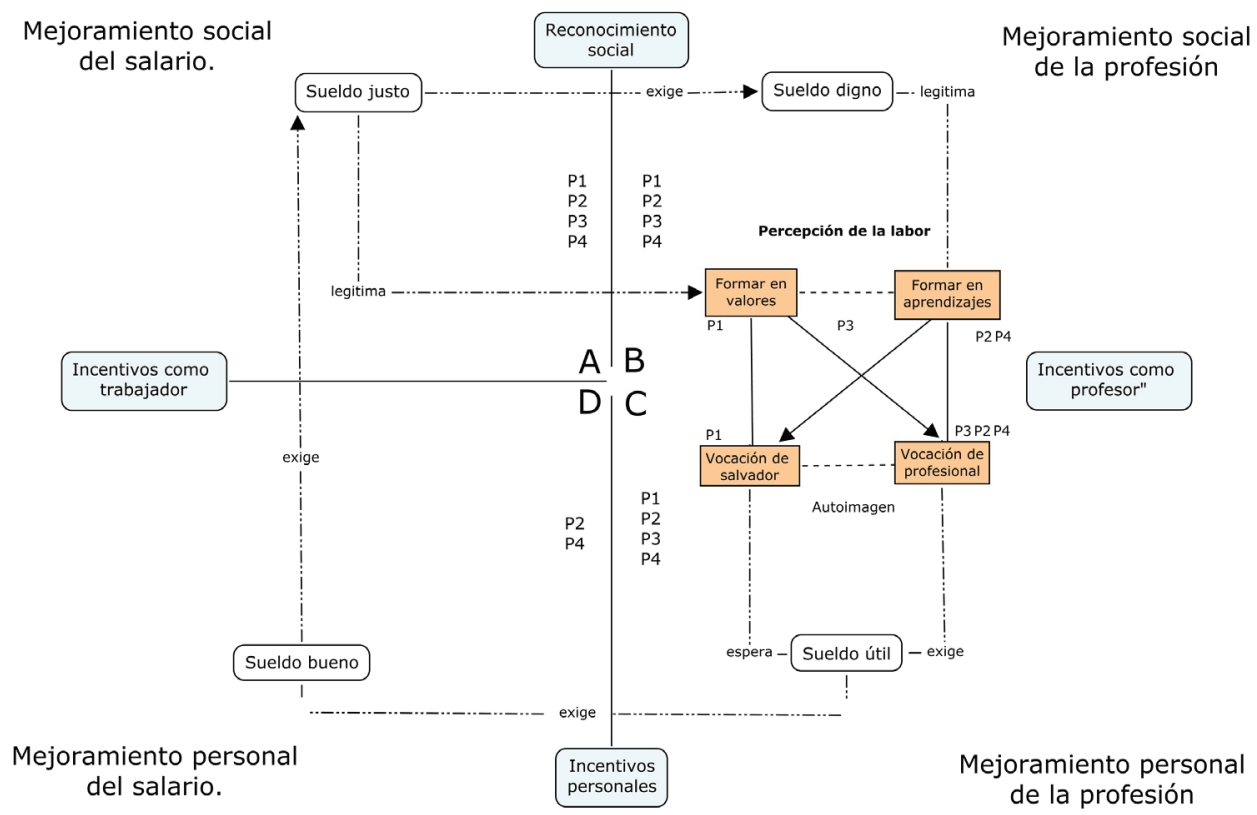

Fuente: elaboración propia.

Lo relevante de este mapa es que permite apreciar que al interior de los núcleos de sentido existen diferencias dependiendo de la percepción de la labor docente que se tenga. Esto permite comprender por qué ciertos discursos de docentes enfatizan elementos de ciertos núcleos, no permitiendo que emerjan elementos de otros núcleos de sentido.

El caso más significativo está en el sueldo justo. Si se aprecia bien, el sueldo justo es un incentivo al buen desempeño que es significado de forma distinta según se tenga una percepción de la labor basada en una vocación de salvador o en una vocación profesional. En el primer caso, el sueldo justo es significado como una evidencia de que el propio trabajo es sólo para valientes, héroes o salvadores. Los sueldos injustos permiten legitimar la pregunta “¿por qué si no estaría aquí?”. En el segundo caso, los docentes significan el sueldo justo como una evidencia de que no se los incentiva como profesionales. Los sueldos injustos generan un discurso en aquellos docentes con vocación profesional que interpelan a la sociedad en general: "¡Sí soy un profesional competente, tienes que pagarme como tal! Mi sueldo es injusto".

El caso del sueldo justo es el más claro, pero sucede también con los otros sueldos. Percibir que hacer bien el trabajo consiste en salvar a los estudiantes (vocación de salvador) se vincula con una interpretación de los incentivos como evidencia empírica de que no están en el trabajo necesariamente por los sueldos o por lo que los otros digan, sino por un llamado

P1: perfil de docentes uno; P2: perfil de docentes dos; P3: perfil de docentes tres; P4: perfil de docentes cuatro. 
interno. Por ello, en sus referencias ni siquiera se enuncia el sueldo bueno que se relaciona al proyecto de vida personal o se enuncia de forma muy secundaria la idea de un sueldo útil. En cambio, percibir que hacer bien el trabajo consiste en ser un profesional (vocación profesional) se vincula con una interpretación de los incentivos como elementos mínimos y completamente exigibles para realizar bien su trabajo. Por lo mismo, el discurso se vuelve más activo y crítico. Es una demanda por mejores condiciones salariales y profesionales.

\section{DISCUSIÓN Y CONCLUSIONES}

\subsection{APORTES AL DEBATE NACIONAL SOBRE LOS PROGRAMAS DE EVALUACIÓN Y LOS INCENTIVOS ECONÓMICOS INDIVIDUALES}

Un primer hallazgo de esta investigación son los núcleos de sentido del mapa conceptual $\mathrm{N}^{\circ}$ 1, que me permiten discutir los supuestos teóricos que sustentan los programas de evaluación del desempeño y los incentivos económicos individuales. Como ya expuse, en Chile, la estabilidad en los resultados de aprendizajes observados vía SIMCE a principios de la década del 2000 se explicó por la lógica económica, por el bajo desempeño de los docentes, y la desconexión de las remuneraciones de éstos con su desempeño efectivo (Mizala y Romaguera 2002a, 2002b). Los núcleos de sentido construidos permiten afirmar que los supuestos teóricos de los programas de incentivo económico individual están operando en uno de los cuatro núcleos: el mejoramiento personal del salario. Las referencias asociadas a recibir un buen salario, esto es, un salario considerado por cada docente como adecuado para su proyecto de vida y que distinga por nivel de desempeño sin problemas, es importante para algunos docentes.

De esta forma, si la atribución de sentido de los programas de incentivos económicos individuales se realiza desde este núcleo de sentido, quiere decir que existe una articulación positiva entre la lógica del programa y el sentido que hace a los docentes. El problema central consiste en que éste no es el único núcleo con el cual se atribuye sentido a los incentivos económicos individuales, sino que también se les atribuye sentido con el núcleo mejoramiento social del salario. Éste es un núcleo transversal a los cuatro perfiles, a diferencia del núcleo mejoramiento personal del salario que está presente en los perfiles dos y cuatro de docentes. De esta forma, cuando los docentes atribuyen sentido a los incentivos económicos individuales, hay dos núcleos operando simultáneamente.

La interpretación que se puede hacer de este fenómeno es que, desde la visión de los docentes, las características propias de sus condiciones de trabajo, específicamente sus bajos sueldos en comparación a otros profesionales, hace que el impacto individual que un incentivo económico tiene en la motivación y el estado de ánimo de un docente se mezcle y diluya en el impacto social que un sueldo justo tiene en el reconocimiento social, logrando con esto que la lógica que sustenta los programas de incentivos económicos individuales sea interpretada de una forma mezclada y diluida por parte de los docentes.

Teóricamente esto implica cuestionar la aplicación mecánica de los supuestos de la lógica económica de los programas de incentivos económicos individuales orientados a los docentes, sin considerar el contexto en que se inserta la solución. Es posible que en un grupo profesional que perciba que su sueldo es socialmente justo, un incentivo económico individual logre operar como un mejoramiento personal del salario sin contratiempos y 
para la mayoría de sus miembros, pero en el caso de los docentes chilenos, tal sentido se mezcla y diluye en el mejoramiento social del salario.

Un segundo problema refiere al sentido negativo atribuido por los docentes a los programas de evaluación de su desempeño académico. Los docentes utilizan los núcleos de sentido de reconocimiento social de la profesión y reconocimiento personal de la profesión para señalar la falta de retroalimentación que el actual sistema tiene al no considerar a los pares cercanos y al generar resultados estandarizados, respectivamente. Es decir, existe una completa ausencia del eje incentivo como profesor, que es el principal eje para atribuir sentido a un sistema de evaluación docente, tanto en su dimensión de reconocimiento social como de incentivo personal.

Frente a lo anterior, es posible señalar que los argumentos que el Colegio de Profesores (2010) utiliza tienen sentido en parte. Por un lado, criticar el sistema de evaluación docente basándose en que hay que revisarlo y crear un sistema realmente formativo es coherente con el discurso docente que emerge en esta investigación. Por otro lado, los argumentos utilizados para criticar los programas de incentivos económicos por enfocarse en la competencia entre pares y al interés individual, no hacen sentido al conjunto de los perfiles docentes. De forma explícita, el discurso de los perfiles dos y cuatro de docentes no estaría de acuerdo con estos argumentos.

\subsection{APORTES AL DEBATE NACIONAL SOBRE CULTURA DOCENTE}

Un segundo hallazgo de esta investigación son las diferencias en los incentivos al buen desempeño profesional según la percepción de la buena realización de la labor que expuse en el mapa $\mathrm{N}^{\circ} 3$. Este hallazgo me permite discutir la idea de choque cultural e incoherencia discursiva con la cual la lógica culturalista ha intentado interpretar la resistencia de los docentes hacia las políticas educativas implementadas en Chile desde la reforma educacional.

Iván Núñez (2004) señala que la década del 2000 corresponde a una etapa de transición en la cultura docente: "estamos en una etapa de transición, en que la identidad técnico y funcionario evoluciona rápidamente y sin retorno a identidad profesional" (2004: 14, énfasis del autor). La resistencia de la cultura burocrática funcionaria a la cultura profesional se interpreta a partir del desarrollo de "defensas simbólicas" o una "coraza cultural protectora" (Belleï et al., 1997) contra la cultura profesional, que la reforma educacional ha intentado implementar.

Las nociones de "defensas simbólicas" y de "coraza cultural protectora" de una cultura tradicional se han ligado, fundamentalmente, a que los docentes se identificarían a ellos mismos como trabajadores humanistas desvinculados de lo productivo. Es por ello que el trabajo de Belleï et al. (1997), referido al Premio de Excelencia Pedagógica, señala que para los docentes: (i) lo prioritario y "real" es el problema contingente de los bajos sueldos y no un premio económico, y (ii) el sentido gremialista de la profesión se siente amenazado en su identidad por la evaluación individual del desempeño.

La argumentación precedente permite justificar dos elementos: i) la hipótesis del choque y resistencia cultural, y ii) la interpretación de que el discurso docente es contradictorio, pues al mismo tiempo que rechazan las políticas de la reforma porque el problema real de ellos son los bajos sueldos y la insatisfacción económica, niegan que lo económico sea el motor de su profesión (Belleï, 2001a). 
Es interesante notar cierta coherencia entre la caracterización de las culturas docentes que realizan los autores reseñados y las distintas percepciones de la labor vocación de salvador y vocación profesional. Pareciera que el perfil uno de docentes posee un discurso coherente con lo que la lógica culturalista denomina una cultura burocrática funcionaria, mientras que los perfiles dos y cuatro poseen un discurso coherente con la cultura profesional. Sin embargo, los argumentos utilizados para esgrimir un choque o resistencia de la cultura burocrática funcionaria con la lógica de los programas de evaluación del desempeño y, sobre todo con los programas de incentivos económicos individuales, puede ser puesta en entredicho, al igual que la interpretación de un discurso contradictorio al interior del cuerpo docente.

En primer lugar, para el caso del programa de evaluación docente, lo más evidente sería encontrar diferencias entre el perfil uno (o lo que los autores llamarían una (cultura burocrática funcionaria) y los perfiles dos y cuatro de docentes (o lo que los autores llamarían una cultura profesional). Por ello, llama la atención que no existan grandes diferencias. Los tres perfiles rechazan la evaluación docente no por la lógica misma de evaluar el trabajo docente, sino por los procedimientos de evaluación. La principal diferencia es que los perfiles dos y cuatro de docentes son capaces de indicar elementos positivos del proceso, pero en ambos casos el rechazo no es a una lógica de evaluación individual, sino a la forma concreta de aplicación de la evaluación.

En segundo lugar, para el caso de los programas de incentivos económicos individuales, es coherente que los perfiles dos y cuatro (o lo que los autores llamarían una cultura profesional) manifiesten el núcleo de sentido del mejoramiento personal del salario. Este núcleo vincula el desempeño con las remuneraciones, justamente porque como el docente sabe que hace bien su trabajo, espera tener mejores condiciones para realizarlo aún mejor. Esto implica una búsqueda de interés personal y una maximización de la ganancia en función de un proyecto personal, que es, a su vez, profesional. Como ya he indicado, es lo bajo del premio lo que critican, no la lógica del premio. Si siguiéramos la línea argumentativa de los autores de la lógica culturalista, sería esperable encontrar un rechazo manifiesto por parte del perfil uno (o lo que los autores llamarían una cultura burocrática funcionaria) a esta lógica, pero lo que ocurre es que el mejoramiento personal del salario no emerge como núcleo de sentido en este perfil.

Un argumento plausible, siguiendo la tesis de estar en una "etapa de transición" de una cultura a otra esgrimida por Núñez (2004), es que ha pasado el tiempo y, hoy por hoy, se ha logrado fortalecer la profesión docente. Por eso en mi investigación encuentro ambos discursos. Éste sería un argumento coherente con la lógica culturalista y, por cierto, no deja de tener sentido.

Ahora bien, la ausencia de crítica en el perfil uno, tanto a la lógica de la evaluación del desempeño como a la lógica de los incentivos económicos individuales, así como la presencia de elementos vocacionales en los perfiles dos y cuatro, me conduce a intentar un nuevo camino interpretativo: no es que exista un rechazo, un choque o una resistencia de la cultura docente burocrática funcionaria a los principios de una cultura profesional basada en una racionalidad instrumental, sino que la lógica culturalista entiende la cultura como una entidad discreta, generando distinciones dicotómicas como humanismo desvinculado de lo productivo v/s profesionalismo interesado en lo productivo. Esta noción de cultura no permite observar matices en la forma de comprender el mundo de los docentes, pues se articula en función de una suma de rasgos discretos y diferenciales, buscando poder 
clasificar. Esto permite hablar de choque e incoherencias, pues se toma a la cultura como un objeto en el mundo, más que una forma de comprender el mundo.

La forma más clara de entender esto es preguntarle a la lógica culturalista cómo interpretaría el sueldo justo del mapa conceptual $\mathrm{N}^{\circ}$ 3. Si seguimos su línea argumentativa, tendríamos que decir que el sueldo justo evidencia una incoherencia, pues mientras es exigido como relevante para el trabajo profesional docente, es indicado como un elemento que no es significativo para el trabajo profesional docente. La noción de cultura basada en rasgos discretos no permite interpretar este hallazgo, porque no es posible que un elemento sea clasificado en la cultura profesional y en la burocrática funcionaria al mismo tiempo, esto genera la idea de choque, tránsito e incoherencia.

Ahora, siguiendo a Geertz (2005), si entendemos por cultura docente una forma de comprender el mundo basada en una experiencia de mundo, entendemos que la percepción que los docentes tienen de la buena realización de su labor genera diferencias en la comprensión de su mundo, lo que no necesariamente nos lleva a hablar de choques y menos de incoherencias. La percepción de la labor, como señala Kelchtermans (2005), es significativa ya que refleja creencias y normas profundamente sostenidas por los docentes sobre el sentido de su trabajo, es decir, refleja formas de comprender el mundo por parte de los docentes.

Esta investigación permite sustentar la idea de que existen al menos tres discursos distintos que construyen formas diferentes de comprender el mundo por parte de los docentes. En primer lugar, existe una percepción de salvador que se orienta a enseñar valores (perfil 1). Esta forma de comprender el trabajo profesional docente, como se aprecia en el mapa conceptual $\mathrm{N}^{\mathrm{o}} 3$, significa el sueldo injusto como una evidencia de que es un salvador, ¿por qué si no, un profesional aceptaría trabajar en las condiciones de injusticia en las que se desenvuelve? En su comprensión del mundo, un salvador o héroe, para enseñar valores, no necesita de un sueldo justo, tampoco de un sueldo útil. Sólo basta con la vocación salvadora. El sueldo digno y bueno, es decir, dos elementos centrales de los núcleos que incentivan el trabajo profesional docente, no son significados y, por ende, no aparecen en el discurso.

En segundo lugar, existe una percepción de profesional que se orienta a enseñar valores (perfil 3). Esta forma de comprender el trabajo profesional docente señala como significativos el sueldo injusto, el sueldo útil y el sueldo digno. En esta comprensión del mundo, lo relevante es que un profesional, para enseñar valores, necesita de sueldos acordes a un trabajo profesional, es decir, un sueldo justo y digno. También precisa de un sueldo útil, pero la no existencia de este sueldo útil refleja que la enseñanza en valores es posible de lograr mediante una buena disposición por parte del docente. El sueldo bueno no es relevante pues los valores del docente siempre van consigo, son inherentes a él o ella.

Por último, existe una percepción profesional que se orienta a enseñar aprendizajes significativos (perfil 2 y 4). Esta forma de comprender el trabajo profesional docente significa como relevantes los cuatro sueldos. En esta comprensión del mundo, lo relevante es que el profesional, para enseñar aprendizajes significativos, necesita de los cuatro sueldos. Por lo mismo, es el discurso más activo y que exige todos los sueldos. Un verdadero profesional requiere de todos los sueldos para hacer bien su trabajo.

Las culturas, entendidas como formas de experienciar y por tanto de comprender el mundo, y no como un mero conjunto de rasgos discretos que uno puede identificar y clasificar, permite entender que vocación e interés hay en todos los discursos identificados 
en esta investigación. Lo relevante no es la presencia o ausencia de rasgos que caracterizan a una u otra cultura, sino las formas de comprender elementos significativos de su mundo. El docente salvador tiene que legitimar su forma de comprender su trabajo: “¿por qué si no estaría aquí?", al igual que el docente profesional: “¡Si soy un profesional competente, tienes que pagarme como tal!".

En 1997 Belleï et al. concluían que para los docentes lo prioritario y real era el "problema contingente de los bajos sueldos y no un premio económico" (1997: 22). Trece años después, esta investigación permite concluir algo similar, pero con un importante matiz: este discurso está lejos de evidenciar un choque cultural o de ser un discurso contradictorio. Esto es así pues no basta con decir que el problema radica en los bajos sueldos, sino que hay que comprender qué quieren decir los docentes cuando dicen "sueldos": ¿de qué sueldo están hablado? ¿Según qué percepción de la labor? ¿Según qué experiencia laboral?

Esto matiza la aparente contradicción y discute la idea de un choque cultural: más que un choque, parece ser que distintas percepciones de la labor significan de forma diversa los incentivos y los problemas del sueldo. El problema del sueldo, más que evocar discursos contradictorios, evoca la centralidad que para la comprensión del mundo de los docentes tiene este tema, pues independiente de la percepción de la buena realización de la labor, es un elemento clave para legitimar la propia percepción, sea ésta salvadora o profesional.

\section{REFERENCIAS BIBLIOGRÁFICAS}

Ávalos, B., Cavada, P., Pardo, M. y Sotomayor, C. (2010). La profesión docente: Temas y discusiones en la literatura internacional. Estudios Pedagógicos, vol.36 (1), 235-263.

Banco Mundial (1996). Prioridades y estrategias para la educación: Examen del Banco Mundial. Washington D.C.: Banco Mundial.

Belleï, C. (2001a). Notas para debatir sobre la profesión docente en Chile. Documento de trabajo interno del MINEDUC parcialmente publicado en OCDE (2004), Revisión de Políticas Nacionales de Educación: Chile (pp. 47-56). París: OCDE.

. (2001b). El talón de Aquiles de la reforma. Análisis sociológico de la política de los 90 hacia los docentes en Chile. En S. Martinic y M. Pardo (Eds.), Economía política de las reformas educativas en América Latina (pp. 129-146). Santiago: CIDE-PREAL.

Belleï, C., Elgueta, S. y Milessi, C. (1997). Percepción y valoración de los profesores sobre el Premio a la Excelencia Docente. Santiago: MINEDUC.

Bravo, D., Falck, D., González, R., Manzi, J. y Peirano, C. (2008). La relación entre la evaluación docente y el rendimiento de los alumnos: Evidencia para el caso de Chile. Santiago: Centro de Microdatos Universidad de Chile.

Burki, S. y Perry, G. (1998). Más allá del Consenso de Washington: La hora de la reforma institucional. Washington D.C.: Banco Mundial.

Cornejo, R. (2009). Condiciones de trabajo y bienestar/malestar docente en profesores de enseñanza media de Santiago de Chile. Educ. Soc., Campinas, vol.30 (107), 409-426.

Colegio de Profesores. (2010). Carrera Profesional Docente. Propuesta del Colegio de Profesores de Chile. Santiago: Colegio de Profesores. Recuperado el 2 de enero de 2013 desde www. colegiodeprofesores.cl/sites/default/files/pdf/pdf2012/carreraprofesional2010.pdf

Cox, C. (2003). Las políticas educacionales de Chile en las últimas dos décadas del siglo XX. En C. Cox (Ed.), Políticas educacionales en el cambio de siglo. La reforma del sistema escolar en Chile (pp. 19-113). Santiago: Editorial Universitaria.

Day, C. y Gu, Q. (2012). Profesores: Vidas nuevas, verdades antiguas. Una influencia decisiva 
Estudios Pedagógicos XLI, N 1: 7-26, 2015

INCENTIVOS AL TRABAJO PROFESIONAL DOCENTE Y SU RELACIÓN CON LAS POLÍTICAS DE EVALUACIÓN E INCENTIVO ECONÓMICO INDIVIDUAL

en la vida de los alumnos. Madrid: Narcea. (2002). School Reform and Transitions in Teacher Professionalism and Identity. International Journal of Educational Research, vol.37 (8), 677-692.

García-Huidobro, J. E. (2001). Conflictos y alianzas en las reformas educativas. Siete tesis basadas en la experiencia chilena. En S. Martinic y M. Pardo (Eds.), Economía política de las reformas educativas en América Latina (pp. 114-124). Santiago: CIDE-PREAL.

Geertz, C. (2005). La interpretación de las culturas. Barcelona: Gedisa.

Hargreaves, A. (2005a). Profesorado, cultura y postmodernidad. Cambian los tiempos, cambia el profesorado. Madrid: Ediciones Morata. (2005b). Cuando vivimos en una época de test estandarizados es cuando más se requiere hacer una comunidad de aprendizaje. Revista Docencia, (27), 64-69.

Hébert, L. (2011). Tools for Text and Image Analysis: An Introduction to Applied Semiotics. Rimouski: Signo.

Kelchtermans, G. (2005). Teachers' Emotions in Educational Reforms: Self-understanding, Vulnerable Commitment and Micropolitical Literacy. Teaching and Teacher Education, vol.21 (8), 995-1006.

Martinic, S. (1992). Análisis estructural: Presentación de un método para el estudio de lógicas culturales. Santiago: Centro de Investigación y Desarrollo de la Educación.

Miles, M. \& Huberman, M. (1994). Qualitative Data Analysis. An Expanded Sourcebook. $2^{\text {nd }}$ $E d$. London: Sage Publications.

Mizala, A. y Romaguera, P. (2002a). Regulación, incentivos y remuneraciones de los profesores en Chile. Documentos de Trabajo Serie Economía 116. Centro de Economía Aplicada (CEA): Universidad de Chile. (2002b). Evaluación del desempeño e incentivos en la educación chilena. Cuadernos de Economía, vol.39 (118), 353-394.

Núñez, I. (2004). La identidad de los docentes. Una mirada histórica en Chile. Santiago: PIIE. (2003). El profesorado, su gremio y la reforma de los años noventa: Presiones de cambio y evolución de la cultura docente. En C. Cox (Ed.), Políticas educacionales en el cambio de siglo. La reforma del sistema escolar en Chile (pp. 455-517). Santiago: Editorial Universitaria. Santiago: PIIE. (1988). El trabajo docente en los proyectos globales de cambio educativo. 1960-1973.

Murillo, J., González, V. y Rizo, H. (1996). Evaluación del desempeño y carrera profesional docente. Un estudio comparado entre 50 países de América y Europa. Santiago: UNESCO.

Sisto, V. (2012). Identidades desafiadas: Individualización, managerialismo y trabajo docente en el Chile actual. Psykhe, vol.21 (2), 35-46.

Strauss, A. y Corbin, J. (2002). Bases de la investigación cualitativa. Técnicas y procedimientos para desarrollar la teoría fundamentada. Medellín: Editorial Universidad de Antioquia.

UNESCO (1990). Declaración mundial sobre educación para todos y marco de acción para satisfacer las necesidades básicas de aprendizaje. Jomtien: UNESCO.

Vegas, E. (2005). Incentives to Improve Teaching. Lessons from Latin America. Washington D.C.: Word Bank. 